\title{
Interaction and stabilization of X-linked inhibitor of apoptosis by Raf-1 protein kinase
}

\author{
SONG TIAN ${ }^{1}$, RAJSHREE R. MEWANI ${ }^{1}$, DEEPAK KUMAR ${ }^{2}$, BIHUA LI $^{1}$, MALIKA T. DANNER $^{1}$, \\ IMRAN AHMAD $^{3}$, AQUILUR RAHMAN ${ }^{1}$, VICENTE NOTARIO ${ }^{1}$, ANATOLY DRITSCHILO ${ }^{1}$, \\ USHA N. KASID ${ }^{1}$ and PRAFULLA C. GOKHALE ${ }^{1}$
}

\begin{abstract}
${ }^{1}$ Department of Radiation Medicine, Lombardi Comprehensive Cancer Center, Georgetown University Medical Center, Washington DC 20057; ${ }^{2}$ Department of Biological and Environmental Sciences, University of the District of Columbia, Washington DC 20008; ${ }^{3}$ NeoPharm, Inc., Lake Forest, IL 60045, USA
\end{abstract}

Received February 13, 2006; Accepted April 17, 2006

\begin{abstract}
Raf-1 serine/threonine protein kinase plays an important role in cell growth, differentiation and cell survival. Recent reports using $c$-raf-1 gene-knockouts have observed MEK/ERK independent functions of Raf-1 in cell survival and protection from apoptosis. Raf-1 has also been shown to be involved in counteracting specific apoptotic pathways by restraining caspase activation, although the precise mechanism is unknown. XIAP is a potent inhibitor of apoptosis that blocks both the mitochondria and death receptor mediated pathways of apoptosis by directly binding to and inhibiting the initiator and effector caspases. In our efforts to understand the mechanism by which Raf- 1 inhibits caspase activation, we discovered a novel interaction between Raf-1 and XIAP. In this study, we describe the physical interaction between Raf-1 and $\mathrm{XIAP}$ in vitro and in vivo in mammalian cells. We also demonstrate that Raf-1 phosphorylates XIAP in vitro and in vivo. Additionally, Raf-1 prevents XIAP degradation in response to different apoptotic triggers. Our studies identify XIAP as a new substrate of Raf- 1 and provide potentially important insight into mechanisms underlying Raf-1 effects on cell survival.
\end{abstract}

Correspondence to: Dr Prafulla C. Gokhale, Department of Radiation Medicine, Georgetown University Medical Center, Room No. E207, The Research Building, 3970 Reservoir Road, NW, Washington DC 20057, USA

E-mail: gokhalep@georgetown.edu

Abbreviations: DMEM, Dulbecco's modified Eagle's medium; FBS, fetal bovine serum; GFP, green fluorescent protein; GST, glutathione S-transferase; IB, immunoblotting; IMEM, improved minimum essential medium; IP, immunoprecipitation; PAGE, polyacrylamide gel electrophoresis; PCR, polymerase chain reaction; SDS, sodium dodecyl sulfate; XIAP, X-linked inhibitor of apoptosis

Key words: interaction, phosphorylation, Raf-1, stabilization, XIAP

\section{Introduction}

Raf-1 protein kinase, an integral component of the Ras/ Raf/MEK/ERK signaling pathway, has been shown to play an important role in cell growth, differentiation and cell survival $(1,2)$. However, reports using genetic knockouts of the mouse $c$-raf- 1 gene have demonstrated a MEK/ERK independent role for Raf-1 in cell survival and protection from apoptosis $(3,4)$. Studies have linked the anti-apoptotic function of Raf-1 to a re-localization of Raf-1 to mitochondria $(5,6)$. Raf-1 is reported to exert prosurvival effects upstream of cytochrome c release by phosphorylating the pro-apoptotic protein Bad, a Bcl-2 family member (7-9). Raf-1 is known to regulate the anti-apoptotic transcription factor $\mathrm{NF}-\kappa \mathrm{B}$ (10) that has been shown to participate in the transcriptional regulation of IAPs $(11,12)$ as well as c-FLIP, an inhibitor of caspase- 8 activation (13). Raf-1 apparently induces NF-кB activation by degradation of $\mathrm{I} \kappa \mathrm{B}$, an inhibitor of $\mathrm{NF}-\kappa \mathrm{B}$, via MEKK-1, independent of MEK/ERK (14). Raf-1 has also been shown to phosphorylate and inhibit the pro-apoptotic function of the ankyrin-repeat protein Tvl-1 by binding to it (15). Tvl-1 promotes the assembly of pro-apoptotic Apaf-1 complexes, caspase activation and apoptosis (16). Furthermore, the MEK/ERK-independent prosurvival function of Raf-1 may be due to antagonizing the activity of apoptotic signalregulated kinase (ASK1) (17). Recently, Raf-1 was shown to counteract apoptosis by suppressing the activation of mammalian sterile 20-like kinase (MST2) (18). Intriguingly, in Raf-1 deficient macrophages and fibroblasts with normal activation of MEK/ERK and NF- $\mathrm{BB}$, cytochrome c release is not increased $(4,19)$. However, caspase activation by selected apoptotic stimuli such as growth factor deprivation and Fas, is enhanced (4). Thus, Raf-1 appears to be involved in counteracting specific apoptotic pathways by restraining caspase activation, although the precise mechanism is unknown.

Members of Bcl-2 and IAP gene families are two classes of cellular apoptotic inhibitors that oppose the cellular destruction by caspases. Bcl-2 proteins can block only the release of cytochrome c from mitochondria (20), whereas IAPs block both the mitochondria and death receptor mediated pathways of apoptosis by directly binding to and inhibiting the 
initiator and effector caspases (21-24). X-linked inhibitor of apoptosis protein (XIAP) is the widely studied IAP member that binds to and potently inhibits caspase-3, caspase- 7 and caspase- 9 activity $(23,24)$.

In this study, we show that Raf-1 protein kinase can physically interact with XIAP both under in vitro and in vivo conditions in mammalian cells. We further demonstrate that Raf-1 phosphorylates XIAP in vitro and in vivo. Additionally, we also show that Raf-1 prevents XIAP degradation in response to different apoptotic triggers.

\section{Materials and methods}

Cell culture and transfections. MDA-MB 231 breast cancer cells, PC-3 prostate cancer cells and A549 lung cancer cells were grown as a monolayer in improved minimum essential medium (IMEM) supplemented with $10 \%$ FBS, 2 mM glutamine, $100 \mu \mathrm{g} / \mathrm{ml}$ streptomycin and $100 \mathrm{U} / \mathrm{ml}$ penicillin. Human embryonic kidney 293T/17 (ATCC, Manassas, VA, USA) cells and Panc-1 pancreatic cancer cells were grown in Dulbecco's modified Eagle's medium (DMEM) supplemented with $10 \%$ FBS, 2 mM glutamine and penicillin/ streptomycin. Cell cultures were maintained at $37^{\circ} \mathrm{C}$ in an atmosphere containing 5\% carbon dioxide. Transient transfections were carried out with Lipofectamine 2000 (Invitrogen, Carlsbad, CA, USA) in HEK 293T/17 cells for $48 \mathrm{~h}$ as per the manufacturer's instructions.

Plasmid construction. DNA inserts for plasmid constructs were amplified by the polymerase chain reaction (PCR) using the AccuPrime Taq DNA Polymerase High Fidelity PCR system (Invitrogen) and primers linked to restriction enzyme sites suitable for cloning. Inserts were sequenced to rule out possible mutations introduced by PCR.

Wild-type Raf-1 expression vector (pCMV-Raf-1) and dominant negative Raf-1 expression vector (Raf-1-S621A) were purchased from BD Biosciences (Palo Alto, CA, USA). Kinase inactive Raf-1 mutant (Raf-1-K375M) was generated using the PCR-based QuikChange ${ }^{\text {TM }}$ site-directed mutagenesis kit (Stratagene, La Jolla, CA, USA) following the manufacturer's instructions. Constitutively active Raf-1 mutant (Raf-1-BXB) in pSR $\alpha$ expression vector was kindly provided by Dr Michael White (The University of Texas, Southwestern Medical Center, Dallas, TX, USA). His-Xpresstagged-Raf-1 was generated by PCR using pCMV-Raf-1 as template and cloned into pcDNA4/HisMAX TOPO vector (Invitrogen).

cDNA fragment encoding the open reading frame of XIAP was amplified by PCR using XIAP cDNA (Image clone \#5532247; Open Biosystems, Huntsville, AL, USA) as the template and cloned into the green fluorescent protein (GFP) expression vector pcDNA3.1/NT-GFP-TOPO or pcDNA4/HisMAX TOPO according to the instructions (Invitrogen) to generate GFP-tagged-XIAP and His-Xpresstagged XIAP, respectively. The mammalian expression plasmid encoding GST-tagged XIAP (pEBG-XIAP) was obtained as a gift from Dr Colin Duckett (University of Michigan, Ann Arbor, MI, USA).

For in vitro studies, bacterial expression plasmid was made by cloning XIAP open reading frame into the pGEX-
4T-3 vector (Amersham Biosciences, Piscataway, NJ, USA). XIAP insert construct was generated by PCR and cloned into the BamHI and EcoRI sites in pGEX-4T-3.

For the mammalian two-hybrid assays, pM-XIAP and pVP16-Raf-1 plasmids were constructed. XIAP insert was generated by PCR and cloned into the EcoRI and BamHI sites in pM vector (BD Biosciences). Raf-1 insert was generated by PCR and cloned into the EcoRI and BamHI sites in pVP16 vector (BD Biosciences).

Co-immunoprecipitation assays. Transfected and untransfected cells were lysed in buffer containing $500 \mathrm{mM}$ HEPES, $\mathrm{pH} 7.2,1 \% \mathrm{NP}-40,10 \%$ glycerol, $5 \mathrm{mM}$ sodium orthovanadate, $1 \mathrm{mM}$ phenylmethylsulfonyl fluoride, $20 \mu \mathrm{g} / \mathrm{ml}$ aprotinin, and $20 \mu \mathrm{g} / \mathrm{ml}$ leupeptin. In addition, we also performed co-immunoprecipitation using NP-40 lysis buffer (25) (10 mM HEPES, pH 7.2, $142.5 \mathrm{mM} \mathrm{KCl,} 5 \mathrm{mM} \mathrm{MgCl}_{2}$, $1 \mathrm{mM}$ EGTA, $0.2 \%$ NP-40, $1 \mathrm{mM}$ PMSF, $5 \mu \mathrm{g} / \mathrm{ml}$ leupeptin and $5 \mu \mathrm{g} / \mathrm{ml}$ aprotinin) or RIPA lysis buffer $(9.1 \mathrm{mM}$ dibasic sodium phosphate, $1.7 \mathrm{mM}$ monobasic sodium phosphate, $150 \mathrm{mM} \mathrm{NaCl}, 1 \% \mathrm{NP}-40,0.5 \%$ sodium deoxycholate, $0.1 \%$ SDS, $1 \mathrm{mM}$ PMSF, $20 \mu \mathrm{g} / \mathrm{ml}$ aprotinin, $20 \mu \mathrm{g} / \mathrm{ml}$ leupeptin and $5 \mathrm{mM}$ sodium orthovanadate). The cell extracts were clarified by centrifugation and used for immunoprecipitation with various antibodies and protein A/G-Agarose (Roche, Indianapolis, IN, USA). As a control we used normal mouse or rabbit IgG (Sigma, St. Louis, MO, USA). Immune complexes were washed three times with lysis buffer, once with $0.5 \mathrm{M} \mathrm{LiCl}, 100 \mathrm{mM}$ Tris-HCl, $\mathrm{pH} 7.4$, and once with $10 \mathrm{mM}$ Tris-HCl, pH 7.4, and resolved on SDS-PAGE. For Western blot analysis, following antibodies were used: monoclonal anti-Raf-1 antibody (BD Biosciences), polyclonal anti-Raf-1 antibody (Santa Cruz, Biotechnology, CA, USA), monoclonal anti-XIAP antibody (BD Biosciences), polyclonal anti-XIAP antibody (Cell Signaling, Beverly, MA, USA), monoclonal and polyclonal anti-GFP antibody (BD Biosciences), monoclonal anti-GST antibody (Novagen, San Diego, CA, USA) and monoclonal anti-Xpress antibody (Invitrogen). The proteins were detected using an enhanced chemiluminescence assay system (Amersham Biosciences).

Production and purification of GST-XIAP. Expression plasmid pGEX-XIAP is described above. pGEX-XIAP and pGEX-4T-3 were transformed into E. coli BL21 (Amersham Biosciences). The protein expression was induced at $25^{\circ} \mathrm{C}$ with $0.5 \mathrm{mM}$ isopropyl-1-thio- $\beta$-D-galactopyranoside for $2 \mathrm{~h}$. Protein extracts were made from the induced cultures by sonication as described in the product manual (Amersham Biosciences). The GST and GST-XIAP proteins were affinity purified using Glutathione Sepharose 4 FastFlow beads (Amersham Biosciences), eluted with glutathione elution buffer (Amersham) and dialyzed in $50 \mathrm{mM}$ Tris $(\mathrm{pH} \mathrm{8.8)}$ with $50 \mathrm{mM} \mathrm{NaCl}$ and $20 \mathrm{mM}$ ß-mercaptoethanol using Float-A-Lyzer (Spectrum Laboratories, Rancho Dominguez, CA, USA).

In vitro binding assay. In vitro translated $\left[{ }^{35} \mathrm{~S}\right]-\mathrm{L}-m e t h i o n i n e-$ labeled Raf-1 protein was prepared from pcDNA4/HisMAXRaf-1 template using the TNT Coupled Reticulocyte Lysate Systems (Promega Corporation, Madison, WI, USA) according 
to the manufacturer's instruction. $\left[{ }^{35} \mathrm{~S}\right]-\mathrm{L}-$ methionine-labeled Raf-1 protein was incubated with GST or GST-XIAP bound to Glutathione Sepharose 4 FastFlow beads for $3 \mathrm{~h}$ at $4^{\circ} \mathrm{C}$. The beads were washed three times with PBS containing protease inhibitor cocktail (Roche) and analyzed by SDS-PAGE and autoradiography.

Mammalian two-hybrid assay. For in vivo protein-protein interaction studies, the BD Matchmaker Mammalian TwoHybrid Assay system (BD Biosciences) and HEK 293T/17 cells were used. XIAP and Raf-1 open reading frames were cloned into mammalian two-hybrid vectors pM and pVP16, respectively, as described above. HEK 293T/17 cells in 6-well tissue culture plates were transfected with $2.0 \mu \mathrm{g}$ pM-XIAP and pVP16-Raf-1 constructs, with $0.4 \mu \mathrm{g}$ of the reporter construct pG5SEAP according to the manufacturer's protocol. Reporter gene assay was performed $36 \mathrm{~h}$ after transfection using GreatEscAPe SEAP assay kit (BD Biosciences) as per the manufacturer's protocol.

Immunofluorescence microscopy. MDA-MB 231, PC-3 and HEK 293T/17 cells were seeded in Lab-Tek II chamber slide (Nalge Nunc International) and allowed to attach overnight. HEK 293T/17 cells were transiently transfected with pcDNA4/HisMAX-Raf-1 and pEBG-XIAP for $48 \mathrm{~h}$. Cells were fixed in $4 \%$ paraformaldehyde ( $\mathrm{pH} 7.4$ ), and permeabilized with $0.1 \%$ Triton X-100 in PBS. Cells were blocked with $2 \%$ BSA and incubated with primary antibodies for $1 \mathrm{~h}$. The cells were then washed with PBS and incubated with secondary antibody in $2 \%$ BSA/PBS along with $2 \mu \mathrm{M}$ TO-PRO-3 reagent (Molecular Probes) for nucleic acid staining. The slides were then washed with PBS and mounted with anti-fade medium (Molecular Probes). Cells were visualized using Olympus FluoView FV300 confocal laser scanning biological microscope.

In vitro kinase assay. Purified GST-XIAP or GST proteins $(5 \mu \mathrm{g})$ were incubated with $0.1 \mu \mathrm{g}$ of active Raf-1 (Upstate, Charlottesville, VA, USA) in kinase assay buffer (Upstate) together with $10 \mathrm{mCi}{ }^{32} \mathrm{P}$-ATP at $30^{\circ} \mathrm{C}$ for $30 \mathrm{~min}$. The reaction was terminated by adding $6 \mathrm{X}$ SDS sample buffer and boiling for $3 \mathrm{~min}$. The samples were separated by SDS-PAGE and the gel dried using gel drying solution (Invitrogen) and analyzed by autoradiography.

In vivo phosphorylation assay. HEK 293T/17 cells were transfected with GST-XIAP together with Raf-1-BXB, Raf-1S621A or control vector. Cells were serum starved overnight and labeled with $\left[{ }^{32} \mathrm{P}\right]$-orthophosphate $(0.5 \mathrm{mCi} / \mathrm{ml})$ in phosphate-free DMEM medium for $3 \mathrm{~h}$. GST-XIAP was immunoprecipitated with anti-GST antibody. The immunoprecipitates were separated on SDS-PAGE and the gel dried using gel drying solution (Invitrogen). Phosphorylated XIAP was detected by autoradiography.

\section{Results}

Raf-1 interacts with XIAP. Initially, to examine whether endogenous Raf-1 is associated with endogenous XIAP in mammalian cells, we performed co-immunoprecipitation

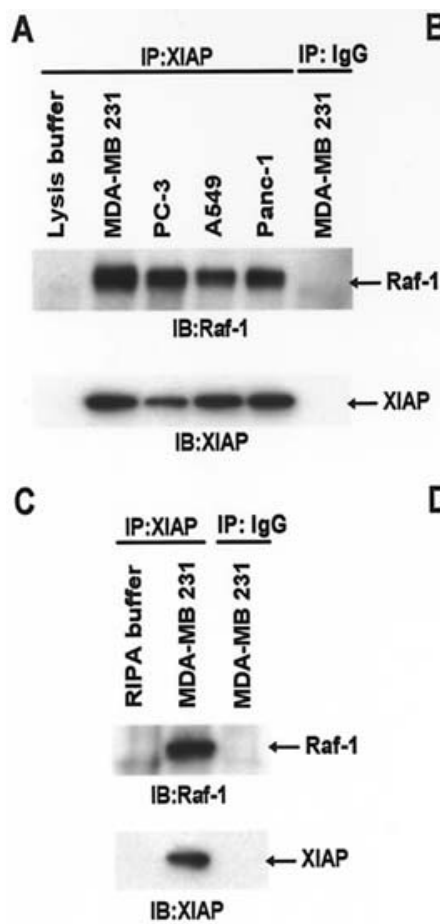

B IP:Raf-1

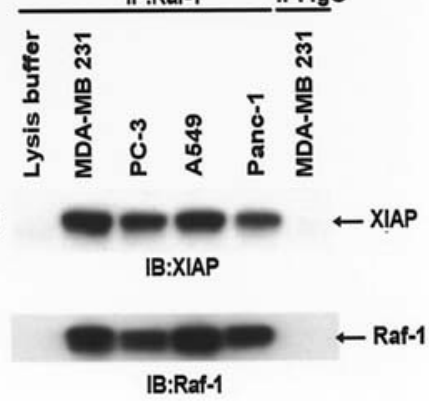

D

Figure 1. Raf-1 protein kinase associates with XIAP in mammalian cells (A) Endogenous XIAP was immunoprecipitated (IP) from various tumor cell extracts using anti-XIAP monoclonal antibody, and the immune complexes were resolved by $8 \%$ SDS-PAGE and co-precipitated Raf-1 was detected by immunoblotting (IB) with anti-Raf-1 polyclonal antibody. Normal mouse $\mathrm{IgG}$ was used as a negative control. The blot was reprobed for XIAP as a loading control. (B) Endogenous Raf-1 was immunoprecipitated using antiRaf-1 polyclonal antibody and resolved by $4-12 \%$ SDS-PAGE and coprecipitated XIAP was detected by immunoblotting with anti-XIAP monoclonal antibody. Normal rabbit IgG was used as a negative control. The blot was reprobed for Raf-1 as a loading control. (C and D) MDA-MB 231 cells were lysed using RIPA lysis buffer. Endogenous XIAP was immunoprecipitated from cell extracts using anti-XIAP monoclonal antibody and coprecipitated Raf-1 was detected by immunoblotting with anti-Raf-1 polyclonal antibody (C). The blot was reprobed for XIAP. Endogenous Raf-1 was immunoprecipitated from MDA-MB 231 cell extracts using anti-Raf-1 polyclonal antibody and co-precipitated XIAP detected by immunoblotting with anti-XIAP monoclonal antibody (D). The blot was reprobed for Raf-1.

experiments. As can be seen in Fig. 1A, Raf-1 could be detected in XIAP immunoprecipitates of various tumor cell extracts but not in control immunoprecipitates, where normal mouse IgG was used. The experiment was also performed in opposite fashion, i.e. Raf- 1 was immunoprecipitated from cell extracts and detected for XIAP by Western blotting (Fig. 1B). In addition, we tested this interaction using salt containing RIPA lysis buffer (Fig. 1C and D) or NP-40 lysis buffer (data not shown) and found comparable results. Further, to establish that the co-immunoprecipitation of XIAP with Raf- 1 is not due to cross reactivity of the antibody, three different Raf-1 antibodies (one monoclonal and two polyclonal) raised against different regions of Raf-1 were used for immunoprecipitation and Western blotting. The results were comparable (data not shown).

To further confirm Raf-1-XIAP interaction, co-immunoprecipitation experiments were performed with transiently transfected epitope-tagged Raf-1 and XIAP plasmid constructs (Fig. 2). For this purpose, we used two constructs of XIAP (GFP-tagged and GST-tagged) and a Raf-1 construct (HisXpress-tagged). First, we transiently transfected HEK 293T/17 

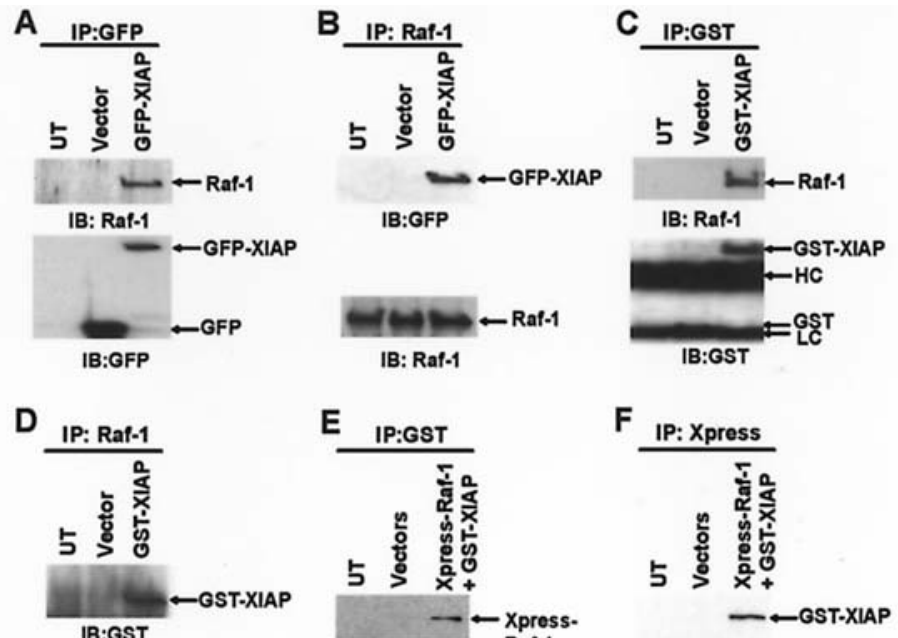

E
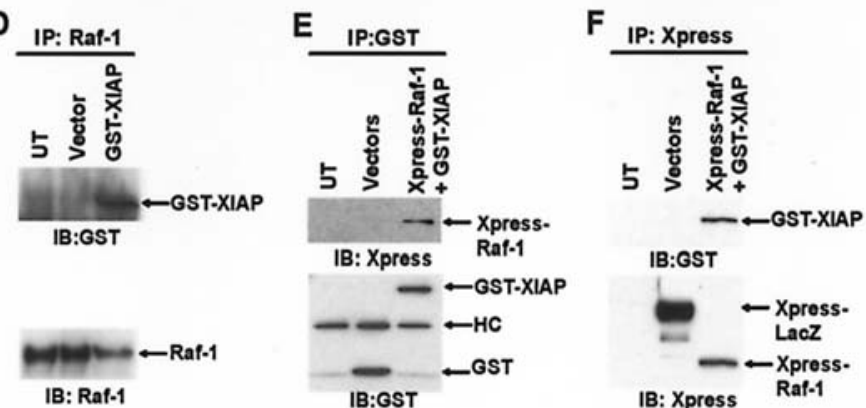

IB:GST

IB: Xpross

G

$\mathrm{H}$
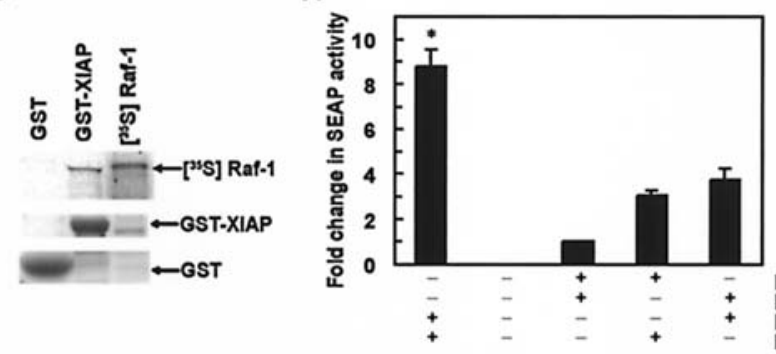

Figure 2. Interaction of Raf-1 with XIAP in vivo and in vitro. (A and B) Reciprocal co-immunoprecipitation of endogenous Raf-1 and exogenous GFP-XIAP. GFP-XIAP or Raf-1 was immunoprecipitated from 293T/17 cells transiently transfected with GFP-XIAP or vector control, followed by immunoblotting with anti-Raf-1 antibody (A, top) and anti-GFP antibody (B, top). UT, untransfected control lysate. The blots were reprobed with anti-GFP antibody (A, bottom) and anti-Raf-1 antibody (B, bottom). (C and D) Reciprocal co-immunoprecipitation of endogenous Raf-1 and exogenous GST-XIAP. GST-XIAP or Raf-1 was immunoprecipitated from 293T/17 cells transiently transfected with GST-XIAP or vector control, followed by immunoblotting with anti-Raf-1 antibody (C, top) and anti-GST antibody (D, top). HC, heavy chain; LC, light chain. The blots were reprobed with anti-GST antibody (C, bottom) and anti-Raf-1 antibody (D, bottom). (E and F) Reciprocal co-immunoprecipitation of exogenous Raf-1 and exogenous XIAP. His-Xpress-Raf-1 or GST-XIAP was immunoprecipitated from 293T/17 cells transiently transfected with both the plasmids or vector control, followed by immunoblotting with anti-Xpress antibody (E, top) and anti-GST antibody (F, top). The blots were reprobed with anti-GST antibody (E, bottom) and anti-Xpress antibody (F, bottom). (G) In vitro binding assay. In vitro translated $\left[{ }^{35} \mathrm{~S}\right]-\mathrm{L}-$ methionine-labeled Raf-1 protein was incubated with GST or GST-XIAP bound to Glutathione Sepharose 4 FastFlow beads for $3 \mathrm{~h}$ at $4{ }^{\circ} \mathrm{C}$. Binding of $\left[{ }^{35} \mathrm{~S}\right]$-L-methionine-labeled Raf-1 to GST-XIAP was detected by washing the beads, followed by $8 \%$ SDS-PAGE and autoradiography. $\left.{ }^{35} \mathrm{~S}\right]-\mathrm{L}-$ methionine-labeled Raf-1 served as marker. (H) Raf-1-XIAP interaction in vivo using the mammalian two-hybrid system. Human embryonic kidney $293 \mathrm{~T} / 17$ cells were co-transfected with $0.4 \mu \mathrm{g}$ of the reporter construct pG5SEAP and $2.0 \mu \mathrm{g}$ of the each plasmid in the combination indicated for $36 \mathrm{~h}$, and the amount of SEAP activity was detected using Great EscAPe SEAP assay kit (BD Biosciences) as per the manufacturer's protocol. Relative fold-change in SEAP activity from three independent experiments is shown (mean $\pm \mathrm{SD}$ ). ${ }^{*} \mathrm{P}<0.001$, compared with other plasmid combinations (Student's t-test).

cells with the GFP-XIAP or GST-XIAP constructs and performed co-immunoprecipitation with endogenous Raf-1. Raf- 1 could be detected in GFP or GST immunoprecipitates of cell extracts transfected with tagged-XIAP constructs and not in the vector transfected cells (Fig. 2A and C). Similar observations were made with Raf-1 immunoprecipitates (Fig. 2B and D). We then carried out transient double transfections of GFP-XIAP and His-Xpress-Raf-1 in HEK 293T/17 cells and performed co-immunoprecipitation experiments. As seen in Fig. 2E and F, exogenous XIAP was found to be associated with exogenous Raf-1. Furthermore, co-immunoprecipitation experiments with COS-1 cells transfected with Raf-1 gave similar results (data not shown). These data confirmed that Raf-1 is associated with XIAP in mammalian cells.
Raf-1 binds with XIAP in vitro. In order to determine whether Raf-1 can bind to XIAP, we performed in vitro binding assay with GST-XIAP and in vitro translated $\left[{ }^{35} \mathrm{~S}\right]-\mathrm{L}-\mathrm{methionine-}$ labeled Raf-1 protein. As a control, we incubated GST protein with the in vitro translated Raf-1 protein. Fig. $2 \mathrm{G}$ shows that Raf-1 can indeed bind to XIAP, but not to control GST protein.

Raf-1 binds to XIAP in vivo in mammalian cells. As a further confirmation on the Raf-1-XIAP interaction, we performed the in vivo mammalian two-hybrid assay. Since this assay is performed in mammalian cells, the results are more likely to represent authentic interaction. In this experiment, XIAP fused to the Gal4 DNA binding domain in pM vector and Raf-1 fused to the activation domain in pVP16 vector were co-transfected in HEK 293T/17 cells along with a reporter 
A
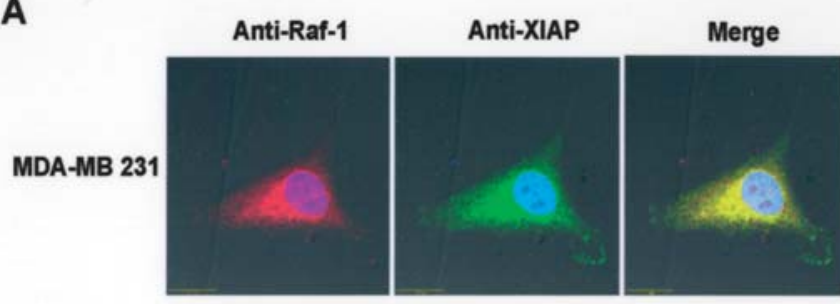

B
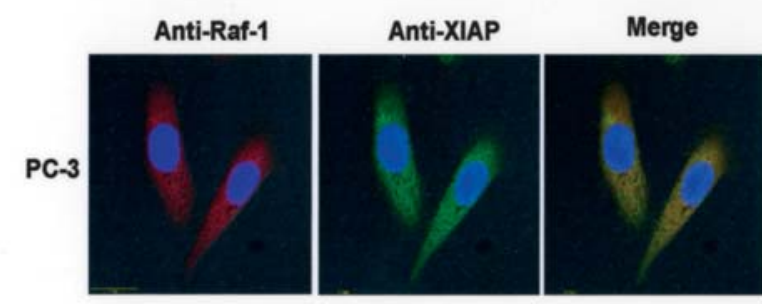

C

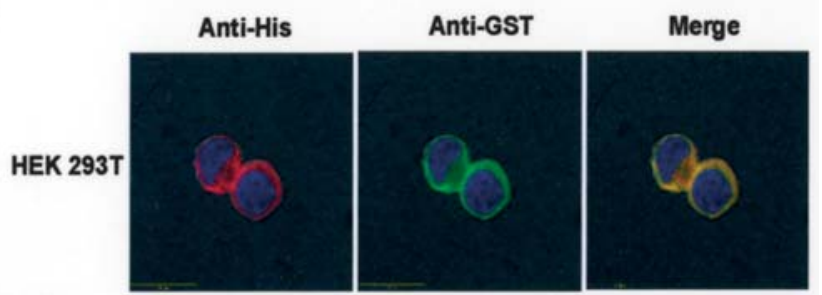

Figure 3. Co-localization of Raf-1 and XIAP in mammalian cells. (A and B) MDA-MB 231 and PC-3 cells were fixed and immunostained with anti-Raf-1 polyclonal antibody and an anti-XIAP monoclonal antibody. (C) HEK 293T/17 cells were transiently transfected with His-Raf-1 and GST-XIAP for $48 \mathrm{~h}$ and the cells were fixed and immunostained with anti-His polyclonal antibdy and anti-GST monoclonal antibody. Alexa Fluor 594-conjugated chicken and antirabbit antibody (Molecular Probes) or Alexa Fluor 488-conjugated chicken anti-mouse antibody (Molecular Probes) was used to virtualize the staining. Nuclear staining was achieved with TO-PRO-3 reagent. Superimposition of the two images (merge) confirms co-localization in yellow.
A

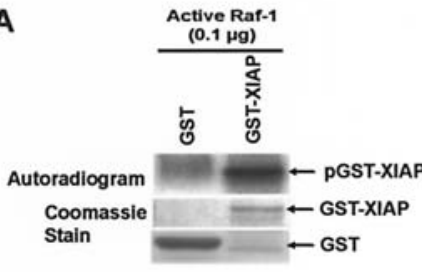

B

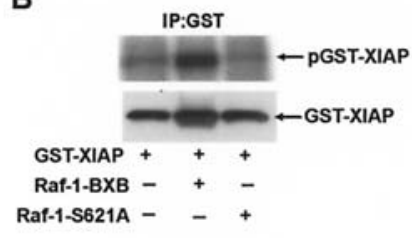

Figure 4. Phosphorylation of XIAP by Raf-1. (A) In vitro phosphorylation of GST-XIAP by active Raf-1. Five $\mu \mathrm{g}$ of purified GST-XIAP and GST proteins were incubated with $0.1 \mu \mathrm{g}$ of active Raf-1 (Upstate) in kinase buffer containing $\left[\gamma_{-}{ }^{32} \mathrm{P}\right]$-ATP. This was followed by SDS-PAGE, autoradiography and coomassie blue stain. (B) Raf-1 phosphorylates XIAP in vivo. HEK 293T/17 cells were transfected with indicated expression constructs, labeled with $\left[{ }^{32} \mathrm{P}\right]$-orthophosphate, and immunoprecipitated with anti-GST antibody. The immunoprecipitates were separated by SDS-PAGE, autoradiographed (top panel), transferred to membrane and Western blotted with antiXIAP antibody (bottom panel).

plasmid pG5SEAP encoding secreted alkaline phosphatase (SEAP). Raf-1-XIAP interaction resulted in the production of high levels of SEAP activity (Fig. 2F). Control experiments, which included co-transfecting pM plasmid together with pVP16 plasmid, pM along with pVP16-Raf-1 or pVP16 along with pM-XIAP, showed significantly lower reporter activity $(\mathrm{P}<0.001)$. Thus, Raf-1 can interact with XIAP in vivo.

Raf-1 co-localizes with XIAP in mammalian cells. We carried out immunofluorescence experiments in tumor cells (MDAMB 231 and PC-3) and in HEK 293T/17 cells transfected with epitope-tagged Raf-1 and XIAP constructs. Fig. 3 shows that Raf-1 and XIAP often co-localize in the cell. In tumor cells, co-localization was performed on endogenous Raf-1 and XIAP proteins using antibodies specific for Raf- 1 and XIAP. Additionally, we performed co-localization studies with exogenously transfected His-Xpress-tagged Raf-1 and GSTtagged XIAP using antibodies specific for His and GST.

Raf-1 kinase phosphorylates XIAP in vitro and in vivo. To investigate whether the physical association of Raf-1 and XIAP results in phosphorylation of XIAP by Raf-1, in vitro and in vivo phosphorylation assays were performed. In vitro kinase assays were performed using purified GST-tagged XIAP as a substrate of Raf-1. In a direct in vitro kinase assay, followed by SDS-PAGE and autoradiography, active Raf-1 was observed to phosphorylate GST-XIAP, while no phosphorylation was observed with purified GST protein (Fig. 4A).

To further examine whether Raf-1 can phosphorylate XIAP in vivo, HEK 293T/17 cells were co-transfected with GSTXIAP and constitutively active Raf-1 construct (Raf-1-BXB) or dominant negative Raf-1 (Raf-1-S621A) construct. After $36 \mathrm{~h}$ of transfection, cells were labeled with $\left[{ }^{32} \mathrm{P}\right]$-orthophosphate for $3 \mathrm{~h}$. SDS-PAGE analysis of GST-XIAP immunoprecipitates showed that constitutively active Raf-1 increase phosphorylation of XIAP as compared with cells transfected with dominant negative Raf-1 and control vector (Fig. 4B).

Raf-1 kinase prevents XIAP degradation in response to apoptotic stimuli. Degradation of XIAP has been shown to be an important mechanism for caspase activation in response to different apoptotic stimuli $(26,27)$. To study whether Raf-1 inhibits XIAP degradation following apoptotic stimuli, we transfected HEK 293T/17 cells with His-XIAP along with various Raf-1 constructs and exposed them to staurosporine treatment or serum starved them. In response to apoptotic stimuli, a significant degradation of XIAP protein expression was observed (Fig. 5A and B). In contrast, XIAP levels were stable in constitutively active Raf- 1 transfected cells. On the other hand, kinase inactive Raf-1 constructs (Raf-1-K375M or Raf-1-S621A) or vector control could not protect XIAP from degradation (Fig. 5A and B). To exclude the involvement of ERK in constitutively active Raf-1 transfected cells, cells were treated with MEK inhibitors (PD98059 and U0126). XIAP levels were found to be stable in Raf-1-BXB transfected cells treated with MEK inhibitors (Fig. 5A and B). Fig. 5C shows significant inhibition of ERK activity following treatment with MEK inhibitors in constitutively active Raf-1 transfected cells. This suggests a direct role of Raf- 1 in XIAP stability. Next we examined whether active Raf-1 stabilized XIAP can suppress caspase-3 activity. In HEK 293T/17 cells treated with staurosporine, caspase-3 activity was markedly decreased by Raf-1-BXB, while kinase inactive Raf-1 mutants or vector control did not inhibit caspase-3 activity (Fig. 5D). 

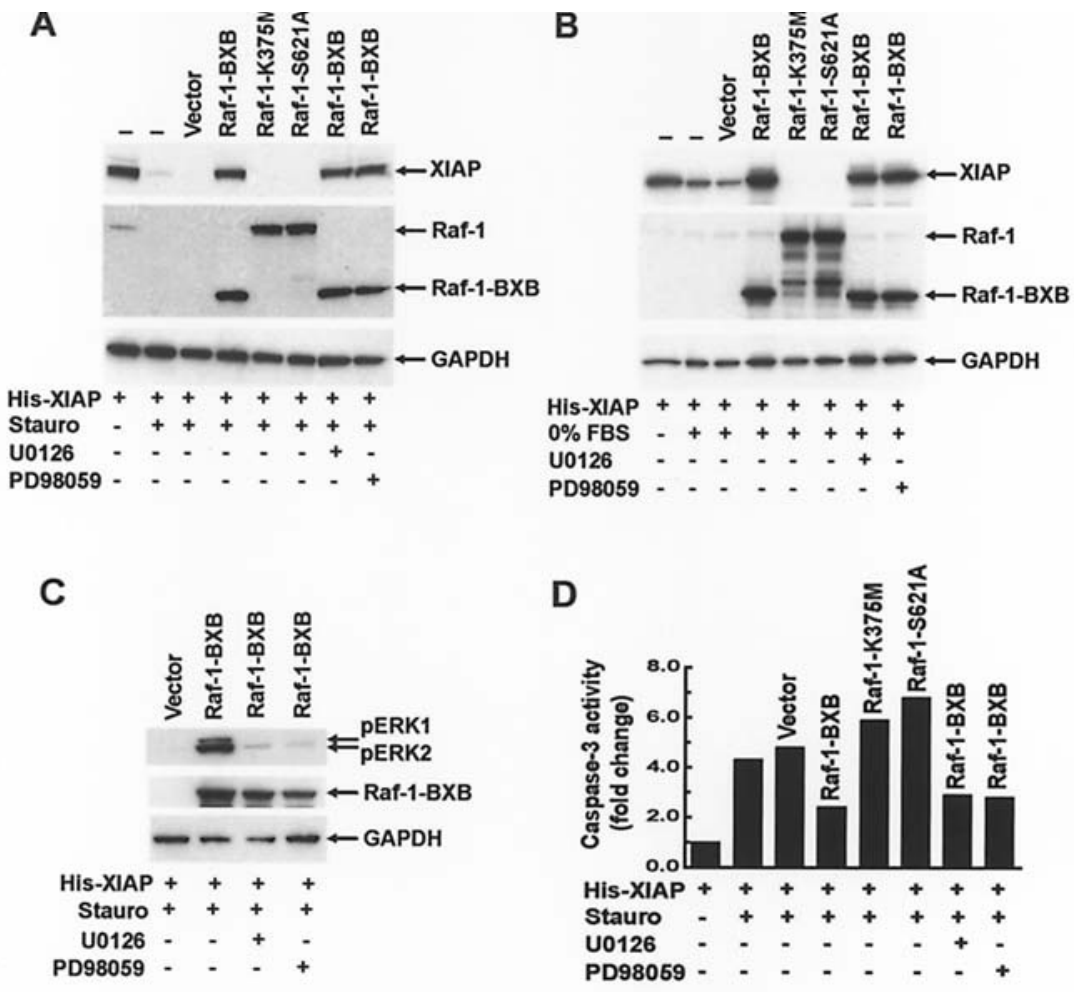

Figure 5. Stabilization of XIAP by Raf-1. (A and B) Constitutively active Raf-1 inhibits XIAP degradation in response to apoptotic stimuli. HEK 293T/17 cells were transfected with indicated expression constructs and treated with $1 \mu \mathrm{M}$ staurosporine for $24 \mathrm{~h}$ (A) or $0 \%$ FBS for $48 \mathrm{~h}$ (B). Cells were lysed and immunoblotted with the indicated antibodies. MEK inhibitors (from Calbiochem), PD 98059 (20 $\mu \mathrm{M})$ and U0126 (4 $\mu \mathrm{M})$ were added to cells along with staurosporine or 0\% FBS treatment. (C) Inhibition of phospho-ERK by MEK inhibitors. HEK 293T/17 cells were transfected with indicated expression vectors and treated with PD 98059 or U0126 as above. Lysates were separated by SDS-PAGE and immunoblotted with anti-phospho-specific ERK antibody (Cell Signaling), anti-Raf-1 antibody and anti-GAPDH antibody. (D) Active Raf-1 stabilized XIAP suppresses caspase-3 activity. HEK 293T/17 cells were transfected with indicated expression constructs and treated with $1 \mu \mathrm{M}$ staurosporine for $6 \mathrm{~h}$. Following treatment, cells were lysed and caspase- 3 activity determined using BD ApoAlert caspase-3 assay kit as per the manufacturer (BD Biosciences). Average fold-change in caspase-3 activity from two experiments is shown.

\section{Discussion}

Raf-1 kinase has been shown to play a central role in cell survival (28). Although, Raf-1 activation of the MEK-ERK cascade has been associated with inhibition of apoptosis, growing evidence also suggests MEK-ERK independent role of Raf-1 in apoptosis suppression and cell survival $(29,30)$. Previously, we and others have demonstrated that antisense raf oligonucleotide mediated inhibition of Raf-1 leads to significant antitumor activity and radiosensitization and chemosensitization of various tumor cell types (31-34). Also, inhibition of Raf-1 is associated with apoptotic cell death as indicated by caspase-3 activation and PARP cleavage $(35,36)$. Raf- 1 exerts pro-survival effects at the premitochondrial levels with inactivation of proapoptotic protein Bad (7-9). However, recent studies have suggested a pro-survival role for Raf-1 at the post-mitochondrial levels $(4,19)$. In our efforts to understand the role of Raf- 1 in inhibiting caspase activation, we discovered a novel interaction between Raf-1 and XIAP in mammalian cells.

Members of the IAP family of proteins, especially XIAP, function as potent inhibitors of caspases $(23,24)$. The IAP families of proteins are characterized by baculovirus IAP repeat (BIR) domains and the RING domain. XIAP contains three BIR domains (24). BIR2 domain of XIAP binds and inhibits caspases-3 and-7, while BIR3 domain inhibits caspase-9. The binding domains between Raf-1 and XIAP remain to be ascertained. The C-terminal RING domain of XIAP has been shown to have ubiquitin protease ligase (E3) activity and is responsible for the autoubiquitination and degradation of XIAP after apoptosis stimuli (22). Here we demonstrate that Raf-1 kinase can phosphorylate XIAP and prevent its degradation in response to apoptotic stimuli. Also, active Raf-1 stabilized XIAP could suppress caspase- 3 activity. These results suggest that XIAP is a new physiological substrate of Raf-1. Recent report has also indicated interaction between chicken IAP homologue ITA and Raf kinases (37) and has proposed a role of Raf at the post-mitochondrial event.

Recently, Akt was also shown to phosphorylate and interact with XIAP (38). XIAP protein sequence contains Akt phosphorylation consensus sequence at residue serine 87 of XIAP (38). It remains to be determined which serine/threonine residues are phosphorylated in XIAP by Raf-1 protein kinase. Previously, B-Raf has been shown to confer protection against apoptosis at the level of caspase activation, downstream of cytochrome c release from mitochondria (39). This suggests that XIAP may also be a relevant target of B-Raf. Future studies are aimed at exploring targeted disruption of Raf-XIAP complex for anti-proliferative properties.

\section{Acknowledgements}

We thank Drs Michael White and Colin Duckett for generously providing the plasmids. This work was supported in part by 
National Institutes of Health Grant (5 PO1 CA074175) and by NeoPharm, Inc. D.K. is supported by MBRS-SCORE grant from NIH. MDA-MB 231, PC-3, A549 and Panc-1 cells were obtained from the Tissue Culture Shared Resource facility of the Lombardi Comprehensive Cancer Center.

\section{References}

1. Kolch W, Heidecker G, Lloyd P and Rapp UR: Raf-1 protein kinase is required for growth of induced NIH/3T3 cells. Nature 349: 426-428, 1991.

2. Magnuson NS, Beck T, Vahidi H, Hahn H, Smola U and Rapp UR: The Raf-1 serine/threonine protein kinase. Semin Cancer Biol 5: 247-253, 1994.

3. Huser M, Luckett J, Chiloeches A, Mercer K, Iwobi M, Giblett S, Sun XM, Brown J, Marais R and Pritchard C: MEK kinase activity is not necessary for Raf-1 function. EMBO J 20: 1940-1951, 2001.

4. Mikula M, Schreiber M, Husak Z, Kucerova L, Ruth J, Wieser R, Zatloukal K, Beug H, Wagner EF and Baccarini M: Embryonic lethality and fetal liver apoptosis in mice lacking the c-raf-1 gene. EMBO J 20: 1952-1962, 2001.

5. Wang HG, Rapp UR and Reed JC: Bcl-2 targets the protein kinase Raf-1 to mitochondria. Cell 87: 629-638, 1996.

6. Zhong J, Troppmair J and Rapp UR: Independent control of cell survival by Raf- 1 and Bcl-2 at the mitochondria. Oncogene 20: 4807-4816, 2001

7. Majewski M, Nieborowska-Skorska M, Salomoni P, Slupianek A, Reiss K, Trotta R, Calabretta B and Skorski T: Activation of mitochondrial Raf-1 is involved in the antiapoptotic effects of Akt. Cancer Res 59: 2815-2819, 1999.

8. Salomoni P, Wasik MA, Riedel RF, Reiss K, Choi JK, Skorski T and Calabretta B: Expression of constitutively active Raf-1 in the mitochondria restores antiapoptotic and leukemogenic potential of a transformation-deficient BCR/ABL mutant. J Exp Med 187: 1995-2007, 1998.

9. Peruzzi F, Prisco M, Dews M, Salomoni P, Grassilli E, Romano G, Calabretta B and Baserga R: Multiple signaling pathways of the insulin-like growth factor 1 receptor in protection from apoptosis. Mol Cell Biol 19: 7203-7215, 1999.

10. Foo SY and Nolan GP: NF-kappa B to the rescue: RELs, apoptosis and cellular transformation. Trends Genet 15: 229-235, 1999.

11. Wang CY, Mayo MW, Korneluk RG, Goeddel DV and Baldwin AS Jr: NF-kappaB antiapoptosis: induction of TRAF1 and TRAF2 and c-IAP1 and c-IAP2 to suppress caspase- 8 activation. Science 281: 1680-1683, 1998.

12. Stehlik C, De Martin R, Kumabashiri I, Schmid JA, Binder BR and Lipp J: Nuclear factor (NF)-kappaB-regulated X-chromosome-linked iap gene expression protects endothelial cells from tumor necrosis factor alpha-induced apoptosis. J Exp Med 188: 211-216, 1998.

13. Micheau O, Lens S, Gaide O, Alevizopoulos K and Tschopp J: NF-kappaB signals induce the expression of c-FLIP. Mol Cell Biol 21: 5299-5305, 2001.

14. Baumann B, Weber C, Troppmair J, Whiteside S, Israel A, Rapp UR and Wirth T: Raf induces NF-kappaB by membrane shuttle kinase MEKK1, a signaling pathway critical for transformation. Proc Natl Acad Sci USA 97: 4615-4620, 2000.

15. Lin JH, Makris A, McMahon C, Bear SE, Patriotis C, Prasad VR, Brent R, Golemis EA and Tsichlis PN: The ankyrin repeatcontaining adaptor protein Tvl-1 is a novel substrate and regulator of Raf-1. J Biol Chem 274: 14706-14715, 1999.

16. Patriotis C, Russeva MG, Lin JH, Srinivasula SM, Markova DZ, Tsatsanis C, Makris A, Alnemri ES and Tsichlis PN: Tpl-2 induces apoptosis by promoting the assembly of protein complexes that contain caspase-9, the adapter protein Tvl-1, and procaspase-3. J Cell Physiol 187: 176-187, 2001.

17. Chen J, Fujii K, Zhang L, Roberts T and Fu H: Raf-1 promotes cell survival by antagonizing apoptosis signal-regulating kinase 1 through a MEK-ERK independent mechanism. Proc Natl Acad Sci USA 98: 7783-7788, 2001.

18. O'Neill E, Rushworth L, Baccarini M and Kolch W: Role of the kinase MST2 in suppression of apoptosis by the proto-oncogene product Raf-1. Science 306: 2267-2270, 2004.
19. Jesenberger V, Procyk KJ, Ruth J, Schreiber M, Theussl HC, Wagner EF and Baccarini M: Protective role of Raf- 1 in Salmonella-induced macrophage apoptosis. J Exp Med 193: 353-364, 2001.

20. Adams JM and Cory S: The Bcl-2 protein family: arbiters of cell survival. Science 281: 1322-1326, 1998.

21. Bratton SB and Cohen GM: Death receptors leave a caspase footprint that Smacs of XIAP. Cell Death Differ 10: 4-6, 2003.

22. Yang Y, Fang S, Jensen JP, Weissman AM and Ashwell JD: Ubiquitin protein ligase activity of IAPs and their degradation in proteasomes in response to apoptotic stimuli. Science 288: 874-877, 2000.

23. Holcik M and Korneluk RG: XIAP, the guardian angel. Nat Rev Mol Cell Biol 2: 550-556, 2001.

24. Salvesen GS and Duckett CS: IAP proteins: blocking the road to death's door. Nat Rev Mol Cell Biol 3: 401-410, 2002.

25. Wang HG, Takayama S, Rapp UR and Reed JC: Bcl-2 interacting protein, BAG-1, binds to and activates the kinase Raf-1. Proc Natl Acad Sci USA 93: 7063-7068, 1996.

26. Yang YL and Li XM: The IAP family: endogenous caspase inhibitors with multiple biological activities. Cell Res 10: 169-177, 2000.

27. Cheng JQ, Jiang X, Fraser M, Li M, Dan HC, Sun M and Tsang BK: Role of X-linked inhibitor of apoptosis protein in chemoresistance in ovarian cancer: possible involvement of the phosphoinositide-3 kinase/Akt pathway. Drug Resist Updat 5: 131-146, 2002.

28. Kolch W: Meaningful relationships: the regulation of the Ras/Raf/MEK/ERK pathway by protein interactions. Biochem J 351: 289-305, 2000.

29. Baccarini M: An old kinase on a new path: Raf and apoptosis. Cell Death Differ 9: 783-785, 2002.

30. Hindley A and Kolch W: Extracellular signal regulated kinase $($ ERK)/mitogen activated protein kinase (MAPK)-independent functions of Raf kinases. J Cell Sci 115: 1575-1581, 2002.

31. Monia BP, Johnston JF, Geiger T, Muller M and Fabbro D: Antitumor activity of a phosphorothioate antisense oligodeoxynucleotide targeted against C-raf kinase. Nat Med 2: 668-675, 1996.

32. Gokhale PC, McRae D, Monia BP, Bagg A, Rahman A, Dritschilo A and Kasid U: Antisense raf oligodeoxyribonucleotide is a radiosensitizer in vivo. Antisense Nucleic Acid Drug Dev 9: 191-201, 1999.

33. Gokhale PC, Zhang C, Newsome JT, Pei J, Ahmad I, Rahman A, Dritschilo A and Kasid UN: Pharmacokinetics, toxicity, and efficacy of ends-modified raf antisense oligodeoxyribonucleotide encapsulated in a novel cationic liposome. Clin Cancer Res 8: 3611-3621, 2002.

34. Mewani RR, Tang W, Rahman A, Dritschilo A, Ahmad I, Kasid UN and Gokhale PC: Enhanced therapeutic effects of doxorubicin and paclitaxel in combination with liposome-entrapped ends-modified raf antisense oligonucleotide against human prostate, lung and breast tumor models. Int J Oncol 24: 1181-1188, 2004.

35. Lau QC, Brusselbach S and Muller R: Abrogation of c-Raf expression induces apoptosis in tumor cells. Oncogene 16: 1899-1902, 1998

36. Slater EP, Stubig T, Lau QC, Achenbach TV, Rapp UR and Muller RC: Raf controlled pathways in the protection of tumor cells from apoptosis. Int J Cancer 104: 425-432, 2003.

37. Rapp UR, Rennefahrt U and Troppmair J: Bcl-2 proteins: master switches at the intersection of death signaling and the survival control by Raf kinases. Biochim Biophys Acta 1644: 149-158, 2004.

38. Dan HC, Sun M, Kaneko S, Feldman RI, Nicosia SV, Wang HG, Tsang BK and Cheng JQ: Akt phosphorylation and stabilization of X-linked inhibitor of apoptosis protein (XIAP). J Biol Chem 279: 5405-5412, 2004.

39. Erhardt P, Schremser EJ and Cooper GM: B-Raf inhibits programmed cell death downstream of cytochrome c release from mitochondria by activating the MEK/Erk pathway. Mol Cell Biol 19: 5308-5315, 1999. 